\title{
Akademische Berufsqualifizierung als konzeptionelle Herausforderung an Hochschulen, Politik und Studierendenforschung
}

Im Rahmen des Bologna-Prozesses wurden in Deutschland Bachelor- und Masterstudiengänge eingeführt, wobei der Bachelor als erster berufsqualifizierender Abschluss die Mehrzahl der Studierenden zu einer Berufstätigkeit führen sollte. Eine ausführliche Diskussion über das Qualifikationsniveau des neuen Abschlusses unterblieb aber genauso wie eine Definition der erwarteten Kompetenzen, was zu Irritationen bei Verantwortlichen in Hochschulen und Unternehmen sowie vielfach zu Zukunftsängsten bei Studierenden geführt hat. Durch erste Studien zum Berufseinstieg von Bachelorabsolventen wird diese Skepsis teilweise bestätigt. Dieser Beitrag gibt einen Überblick über den Diskussionsstand anhand aktueller empirischer Studien. ${ }^{1}$

\section{Einleitung}

Der Anspruch einer stärkeren Orientierung der Hochschulbildung an der beruflichen Praxis ist von Beginn an mit dem Bologna-Prozess verbunden. Die Bologna-Erklärung formuliert als zentrales Vorhaben die Einführung eines Systems leicht verständlicher und vergleichbarer Abschlüsse und verknüpft dies mit dem Ziel, „die arbeitsmarktrelevanten Qualifikationen der europäischen Bürger ebenso wie die internationale Wettbewerbsfähigkeit des europäischen Hochschulsystems zu fördern" (Europäische Bildungsminister 1999). Ferner wird an den Bachelor der Anspruch formuliert, „eine für den europäischen Arbeitsmarkt relevante Qualifikationsebene“ (ebd.) darzustellen. Darüber hinaus nehmen die Europäischen Bildungsminister $^{2}$ (2003) Bezug auf die Lissabon-Strategie, die darauf abzielt, Europa zum ,wettbewerbsfähigsten, wissensbasierten Wirtschaftsraum der Welt" zu machen (Europäischer Rat 2000). In den Dokumenten des Bologna-Prozesses lässt sich eine schrittweise Engführung des Praxisbegriffs auf „Employability“, also auf die reine Beschäftigungsfähigkeit der Absolventen nachzeichnen, wobei der Begriff selbst kaum inhaltlich konkretisiert wird und somit eine "diffuse und mehrdeutige Kategorie" bleibt (Koepernik/Wolter 2010, S. 58). Obwohl beispielsweise mit den Ansätzen der Berufs- und Praxis- orientierung konzeptionelle Alternativen zur „Employability“ bestehen, dominiert dieser Begriff trotz seiner Unschärfe und ohne wahrnehmbare inhaltliche Auseinandersetzung über unterschiedliche Ansätze einer akademischen Berufsqualifizierung die hochschulpolitische Diskussion in Deutschland, was sich unter anderem auf die offensive Zustimmung vonseiten der Arbeitgeber zurückführen lässt.

Um ihre Unterstützung für die Einführung des Bachelorgrades als erstem berufsqualifizierenden Hochschulabschluss deutlich zu machen, gaben Personalverantwortliche von 15 Großunternehmen im September 2004 die Erklärung „Bachelor welcome!“ ab, in der sie sich für eine „konsequente Umstellung“ des Studiensystems auf Bachelor- und Masterabschlüsse stark machten (Bensel et al. 2004). Hierbei forderten sie einen engen Praxisbezug des Studiums und die Einbeziehung der Arbeitgeber in die Wei-

\footnotetext{
1 Ulf Banscherus, Annerose Gulbins, Klemens Himpele und Sonja Staack haben im Auftrag der Max-Traeger-Stiftung und der Gewerkschaft Erziehung und Wissenschaft (GEW) die Potenziale des Bologna-Prozesses und dessen Umsetzung in Deutschland untersucht (Banscherus et al. 2009). Die aktualisierten Ergebnisse dieser Studie fließen in den vorliegenden Beitrag ein.

2 Im Interesse von Einheitlichkeit und Lesbarkeit sowie zur Erfüllung der redaktionellen Vorgaben erfolgen Personenbezeichnungen in diesem Text durchgehend in der grammatisch männlichen Form. Die Aussagen beziehen sich aber in jedem Fall auf alle Angehörigen der jeweiligen Personengruppe, unabhängig von ihrem biologischen oder sozialen Geschlecht.
}

terentwicklung der Qualitätssicherung. 2006 ergänzten 22 Personalverantwortliche in der Erklärung „More Bachelors and Masters welcome!" ihre Forderungen um einige Punkte: die Stärkung der Hochschulautonomie und die Eröffnung neuer Finanzierungswege; empfohlen wird auch die Implementierung unternehmerischer Strukturen an den Hochschulen (Bake et al. 2006).

Ulf Banscherus, Diplom-Politologe,
promoviert im Bereich der Hochschulfor-
schung an der Technischen Universität
Dresden. Arbeitsschwerpunkte: Bildungs-
soziologie, Studienstrukturreform/Bologna-
Prozess, Lebenslanges Lernen an Hoch-
schulen/Durchlässigkeit im Bildungssystem.
e-mail: ulf.banscherus@mailbox.
tu-dresden.de
Klemens Himpele, Diplom-Volkswirt, ist
Referent im Vorstandsbereich Hochschule
und Forschung der Gewerkschaft Erzie-
hung und Wissenschaft (GEW). Arbeits-
schwerpunkte: Bildungsökonomie, Soziale
Ungleichheit im Bildungssystem, Studien-
finanzierung.
e-mail: klemens.himpele@gew.de
Sonja Staack, Diplom-Chemikerin, ist Fach-
referentin für Bildungspolitik im Bundestag
und Mitglied im Bezirksvorstand Berlin der
Vereinten Dienstleistungsgewerkschaft
(ver.di). Arbeitsschwerpunkte: Studienre-
form, Bildungsbeteiligung, Verhältnis von
akademischer und beruflicher Bildung.
e-mail: sonja.staack@hamburg.de




\section{Verbreitete Skepsis bei Studienanfängern}

Die wichtigsten Gründe für die Wahl eines Bachelorstudiums haben sich im Laufe der Zeit verändert, wie die regelmäßigen Befragungen des Hochschulinformationssystems (HIS) unter Studienanfängern zeigen. ${ }^{3}$ Die wichtigsten Ergebnisse sind in Tabelle 1 zusammengefasst.

Während der Anteil derjenigen, die einen Bachelorstudiengang wegen der Option, einen Masterstudiengang anzuschließen, befürworten, sich seit der ersten Befragung im Wintersemester 2000/2001 nur wenig verändert hat, sind die Bedeutung der kürzeren Studienzeit und der internationalen Verbreitung von Bachelor- und Masterstudiengängen als Beweggründe deutlich zurückgegangen. Ein entsprechender Trend lässt sich auch bei der Bewertung der Arbeitsmarktchancen beobachten: Im Wintersemester 2000/2001 wählten noch $60 \%$ der Studienanfänger einen Bachelorstudiengang aufgrund erwarteter positiver Arbeitsmarktchancen, im Wintersemester 2007/2008 waren es nur noch $37 \%$ - wobei dieser Anteil an Fachhochschulen mit $46 \%$ höher, an den Universitäten mit lediglich $31 \%$ Zustimmung dagegen niedriger ausfällt (Heine et al. 2008, S. 172). Die unklaren Arbeitsmarktchancen mit einem Bachelorabschluss bilden weiterhin den wichtigsten Grund für Studienanfänger, sich gegen ein Bachelorstudium zu entscheiden, sofern dies infolge der fortgeschrittenen Verbreitung der gestuften Studienstruktur überhaupt noch möglich ist: $64 \%$ derjenigen, die einen Bachelorstudiengang nicht in Betracht ziehen, geben diese Begründung an - das entspricht $22 \%$ aller Studienanfänger (Krawietz 2007). Auch die Daten des in regelmäßigen Abständen durchgeführten Studierendensurveys der AG Hochschul-

\section{Tabelle 1: Gründe für die Wahl eines Bachelorstudiengangs bei} deutschen Studienanfängern - in \% -

\begin{tabular}{lcc}
\hline & $\begin{array}{c}\text { Wintersemester } \\
\mathbf{2 0 0 0 / 2 0 0 1}\end{array}$ & $\begin{array}{c}\text { Wintersemester } \\
\mathbf{2 0 0 7 / 2 0 0 8}\end{array}$ \\
\hline Möglichkeit der Studienfortsetzung mit einem Master- & 79 & 73 \\
Studiengang & 45 & 29 \\
kurze Studienzeit & 81 & 53 \\
international verbreiteter Studienabschluss & 60 & 37 \\
gute Arbeitsmarktchancen & & WVS MITTEILUNGEN \\
\hline Quelle: Heine et al. 2008, S. 172; eigene Darstellung. & & MS
\end{tabular}

forschung an der Universität Konstanz sind eindeutig: Haben im Jahr 2001 noch $25 \%$ der befragten Studierenden mit dem Bachelor gute Chancen auf dem Arbeitsmarkt verbunden, lag dieser Wert 2007 nur bei $12 \%{ }^{4}$ Damit korrespondiert die Einschätzung, dass der Bachelorabschluss zu Hochschulabsolventen „zweiter Klasse“ führe. Dieser Aussage stimmte mit $52 \%$ die Mehrheit der Befragten zu (Bargel et al. 2008, S. 43).

Hierbei resultieren die Unsicherheiten im Hinblick auf die „Berufstauglichkeit“ der neuen Studiengänge, insbesondere des Bachelorabschlusses, sicherlich teilweise aus den mit der Studienstrukturreform selbst verbundenen allgemeinen Unsicherheiten. So erklärt etwa Klös (2010) diese Unsicherheiten mit den neuen Abschlüssen im Wesentlichen als ein informationsökonomisches Phänomen, das erst mit einer zunehmenden Verbreitung der neuen Abschlüsse überwunden werde. Ein solcher Informationsmangel kann möglicherweise die verbreitete Skepsis gegenüber den neuen Abschlüssen erklären, jedoch nicht den feststellbaren Einbruch des Vertrauens in ihre Vorteile und Arbeitsmarktchancen. Die wachsende Skepsis gegenüber den neuen Abschlüssen ist ohnehin nicht das einzige Problem. Hinzu kommt, dass die Konzeption des Bachelors als Regelabschluss durch Hochschulen und Politik das traditionell zu beobachtende, weitgehende Fehlen einer plausiblen Ausgestaltung der berufsvorbereitenden Aufgaben der Hochschulen weiter verschärft. In der Vergangenheit konnten sich insbesondere die Universitäten unter Hinweis auf die postulierte grundlegende inhaltliche Differenz zwischen Wissenschaft und gabe überwiegend entziehen. Diese Position ist jedoch bei einer verkürzten Studienzeit und einer anders akzentuierten Aufgabenzuschreibung zumindest des Bachelorstudiums nicht weiter aufrecht zu erhalten. Berufsausbildung dieser Definitionsauf-

\section{Bewertung der Abschlüsse auf dem privaten Arbeitsmarkt}

Ein klar definiertes Einstiegslevel auf dem Arbeitsmarkt für Bachelorabsolventen lässt sich aus den vorliegenden Daten bislang nicht ableiten. Bei der Unternehmensbefragung des Instituts der Deutschen Wirtschaft (IW) im Jahr 2004 gaben $38 \%$ der befragten Unternehmen an, Einstiegspositionen für Bachelorabsolventen auf der Ebene von beruflichen Aufstiegsfortbildungen wie etwa der Fachwirt- oder Meisterprüfung anzusiedeln, 30 \% der Unternehmen bieten Bachelorabsolventen Positionen auf der Ebene von traditionellen Hochschulabsolventen an (Konegen-Grenier 2004, S. 12). ${ }^{5}$ Eine Einstufung von Bachelorabsolventen auf dem Niveau von Absolventen einer Berufsausbildung kommt dagegen nur für $7 \%$ der Unternehmen infrage. Die Befürchtung, dass die Bachelorabsolventen diese Gruppe unmittelbar verdrängen und für Absolventen einer Berufsausbildung den Berufseinstieg blockieren könnten, wird durch diese Zahlen folglich nicht bestätigt. Nahegelegt wird allerdings die Befürchtung, dass die Einführung des Bachelors zu einem Bedeutungsverlust des Fortbildungspfades von Nichtakademikern führt und damit einen gerade für kleine und mittelständische Unternehmen traditionell wichtigen Karrierepfad entwertet (vgl. hierzu auch Dobischat et al. 2008). Werner et al. (2008) kommen zu dem Ergebnis, dass dieses Konkurrenzpotenzial in größeren

3 Für die aktuelle HIS-Studienanfängerbefragung wurden im Wintersemester 2007/2008 insgesamt rund 27.800 deutsche Studienanfänger an 71 Hochschulen postalisch angeschrieben, von denen sich 8.342 an der Erhebung beteiligt haben, was einem Rücklauf von ca. $30 \%$ entspricht (Heine et al. 2008, S. 7).

4 Für den aktuellen Studierendensurvey wurden im Wintersemester 2006/2007 insgesamt 28.000 Studierende an 25 ausgewählten Hochschulen postalisch angeschrieben, von denen sich 8.350 an der Erhebung beteiligt haben, was einem Rücklauf von $32 \%$ entspricht (Bargel et al. 2008, S. 66).

5 An der Unternehmensbefragung im Rahmen der Frühjahrsumfrage des IW haben sich im Zeitraum Mai und Juni 2004 insgesamt 672 Unternehmen aus allen Branchen beteiligt. Hierbei wurde eine Rücklaufquote von 14 \% erreicht (Konegen-Grenier 2004, S. 3). 
Unternehmen aufgrund der stärker ausdifferenzierten Hierarchien und fachlichen Zuständigkeiten geringer ausgeprägt zu sein scheint. Kleinere und mittlere Unternehmen (unter 1.000 Beschäftigte) wurden in der zugrunde liegenden Unternehmensbefragung nicht berücksichtigt. ${ }^{6}$ Wie Dobischat et al. weisen sie auf die anhaltende Unsicherheit hinsichtlich der Verortung von Bachelorabsolventen hin.

Um auch beruflich Qualifizierten weiterhin einen Aufstieg durch Fortbildung zu ermöglichen und die Risiken der Einführung der gestuften Abschlüsse an den Hochschulen für das Berufsbildungssystem bereits konzeptionell abzufangen, müsste ihnen folglich der Aufstieg über ein - gegebenenfalls berufsbegleitendes - Studium deutlich erleichtert werden. Habermann/Lohaus (2006) weisen außerdem darauf hin, dass die kürzeren Studiengänge für Abiturienten attraktiv sein dürften, die bisher eine anspruchsvolle duale Berufsausbildung gewählt haben - die Unternehmen müssten in diesem Bereich also mit einem Verlust besonders qualifizierter Bewerber rechnen. Deutliche Unterschiede zwischen den verschiedenen Abschlussarten finden sich - zumindest für den Bereich der Ingenieurwissenschaften - bei den Tätigkeitsfeldern der Absolventen: Während Absolventen eines Masterstudiengangs häufiger in den Bereichen Forschung, Entwicklung und Konstruktion eingesetzt werden, finden sich Bachelorabsolventen häufiger in den Bereichen Produktion, Vertrieb und Montage (Konegen-Grenier/Koppel 2009, S. 7).

Neben den Bedingungen des Berufseinstiegs nach dem Bachelor stellt sich auch die Frage, welche Möglichkeiten der beruflichen Weiterentwicklung den Absolventen offen stehen. Wenn diese die gleichen Chancen haben wollen, Führungspositionen zu erreichen, wie traditionelle Hochschulabsolventen, müssen sie nach Auffassung der Mehrheit der Unternehmen (51 \%) im Verlauf ihrer Karriere einen weiteren Hochschulabschluss erwerben (Konegen-Grenier 2004, S. 15). Diese Zahl ist im Zeitverlauf recht stabil. So stimmten auch in der aktuellen IW-Befragung $49 \%$ der befragten Unternehmen der Aussage $\mathrm{zu}$, dass Bachelorabsolventen in den Ingenieurberufen für eine positive Karriereentwicklung einen Masterabschluss benötigen (Konegen-Grenier/Koppel 2009, S. 9). ${ }^{7}$ Damit sich die Bachelorabsolventen auf dem Arbeitsmarkt nicht mit einer neuen gläsernen Decke konfrontiert sehen, müsste folglich das Absolvieren eines Masterstudiengangs zu einem späteren Zeitpunkt - berufsbegleitend, in Freistellung oder als Erwerbsunterbrechung - von der Ausnahme zu einem üblichen Bildungsweg werden. Die Auseinandersetzung mit neuen Konzepten zur Weiterbildung und Mitarbeiterbindung der neuen Absolventengruppe spielt in den Unternehmen bislang allerdings nur selten eine Rolle (Geighardt 2009). Eine entsprechende Entwicklung stellt zudem neue Anforderungen an die Hochschulen, die sich auf diese Zielgruppen einstellen müssen, sowie auch an die öffentliche Ausbildungsförderung, die sich bislang ausschließlich am Erststudium orientiert.

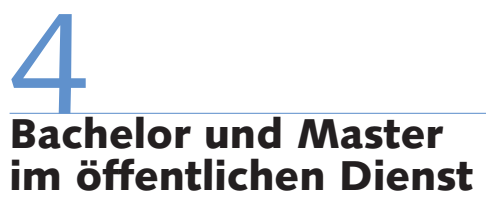

Bei der Akzeptanz des Bachelors auf dem Arbeitsmarkt spielt der öffentliche Dienst aufgrund seiner Vorbildfunktion für die Tarifentwicklung eine besondere Rolle. Hinzu kommt, dass Bund und Länder die Entwicklung der Tarifverträge für den öffentlichen Dienst (TVöD, TV-L) sowie die laufbahnrechtliche Einordnung bestimmter Bildungsabschlüsse unmittelbar beeinflussen und hiermit die Anerkennung des Bachelors als neuen Hochschulgrad forcieren können. Umso bemerkenswerter ist es, dass die Einordnung des Bachelorabschlusses auch im Bereich des öffentlichen Dienstes weiterhin viele offene Fragen aufweist. Seit 2002 werden Bachelorabschlüsse (wie das traditionelle Fachhochschuldiplom) dem gehobenen Dienst zugeordnet, Masterabschlüsse dem höheren Dienst. Eine entsprechende Regelung ist mit dem Gesetz zur Neuordnung und Modernisierung des Bundesdienstrechts (Dienstrechtsneuordnungsgesetz - DNeuG) auch für Beamte getroffen worden. Als Neuerung ist hervorzuheben, dass auch Masterabschlüsse an Fachhochschulen den Zugang zu Laufbahnen des höheren Dienstes ermöglichen können. Mit dem Beschluss der Kultusministerkonferenz (20.09.2007) sowie der Innenministerkonferenz (07.12.2007) zum „Zugang zu den Laufbahnen des höheren Dienstes durch Masterabschluss an Fachhochschulen" wurde allen Absolventen von akkreditierten Fachhochschulmas- terstudiengängen der Zugang zum höheren Dienst eröffnet. Durch die Knüpfung der Zulassung an die Akkreditierung von Fachhochschulmastern wird gleichwohl eine gewisse Ungleichbehandlung von Universitäts- und Fachhochschulstudiengängen aufrechterhalten.

Bachelorabsolventen müssen - wollen sie sich die Möglichkeit eines Aufstiegs in den höheren Dienst bewahren - im Verlauf ihres Berufsweges einen Masterabschluss nachholen. Gegenüber den traditionellen Hochschulabschlüssen dürfte der Bachelor hierdurch an einem gewissen Attraktivitätsdefizit leiden. Die Frage nach der Einstufung des Bachelors könnte sich noch dringender stellen, falls die Hochschulen in den kommenden Jahren stärker auf vierjährige Bachelorprogramme setzen, um die neuen Abschlüsse inhaltlich und qualitativ aufzuwerten - und näher an die Diplomund Magisterabschlüsse heranzuführen. Bislang dominierte in Deutschland eine Orientierung auf sechssemestrige Bachelor (vgl. HRK 2009). Nach dem „BolognaGipfel“ im Juli 2009 erklärte Bundesbildungsministerin Schavan allerdings: „Für die Länge des Bachelorstudiums brauchen wir mehr Flexibilität. Es kann auch erforderlich sein, statt sechs auch sieben oder acht Semester im Bachelorstudiengang zu studieren" (Pressemitteilung des BMBF vom 07.07.2009). Dies würde im Rahmen der bisherigen Laufbahnstruktur bedeuten, dass eine nur geringfügig verkürzte Studienzeit mit einer wesentlichen Einschränkung der Aufstiegschancen einherginge.

\section{Differenzierte Ergebnisse von Absolventenstudien}

Erste Hinweise auf den Verlauf des Übergangs vom Studium in den Beruf bietet die Untersuchung des Absolventenjahr-

6 Für die Bedarfsanalyse wurden sieben Experten in Kurzinterviews sowie ein Dachverband, drei Branchenverbände und zwei bis vier Unternehmen mit mindestens 1.000 Beschäftigten in jeder dieser drei Branchen in ausführlicheren Interviews befragt.

7 Die Datengrundlage hierfür ist eine Sonderauswertung des IW-Zukunftspanels 2009, an dem sich 3.906 Unternehmen beteiligt haben. Für die Betrachtung der Situation von Ingenieuren wurden Daten von 1.753 Unternehmen berücksichtigt. 
Tabelle 2: Ausgewählte Ergebnisse der INCHER-Absolventenstudie 2009 (nur regulär Erwerbstätige) - in \% -

\begin{tabular}{lccc}
\hline & $\begin{array}{c}\text { Sehr gute bis gute } \\
\text { Angemessenheit der } \\
\text { beruflichen Situation } \\
\text { zur Ausbildung }\end{array}$ & $\begin{array}{c}\text { Sehr gute bis gute } \\
\text { Qualifikations- } \\
\text { verwendung }\end{array}$ & $\begin{array}{c}\text { Kein Hochschul- } \\
\text { abschluss erforderlich } \\
\text { für Tätigkeit }\end{array}$ \\
\hline Bachelor FH & 61 & 39 & 12 \\
Master FH & 62 & 57 & 1 \\
Diplom FH & 64 & 45 & 8 \\
Bachelor Uni & 49 & 27 & 21 \\
Master Uni & 63 & 50 & 6 \\
Diplom Uni & 64 & 45 & 16 \\
Magister Uni & 42 & 31 & 8 \\
\hline Insgesamt & 61 & 43 & WS \\
\hline
\end{tabular}

gangs 2007 eineinhalb Jahre nach Studienabschluss, die im Sommersemester 2009 im Auftrag des Bundesministeriums für Bildung und Forschung vom Internationalen Zentrum für Hochschulforschung an der Universität Kassel (INCHER) durchgeführt wurde. An dieser Studie waren 41 Hochschulen beteiligt, an denen im Jahr 2007 rund 99.000 Personen ihren Abschluss gemacht haben, was mehr als einem Drittel aller Absolventen in Deutschland entspricht. Trotz der Beteiligung von mehr als 37.000 Absolventen müssen die Ergebnisse dennoch als vorläufig betrachtet werden, da der Anteil der Daten von Absolventen von Bachelor- und Masterstudiengängen mit ca. 10 bzw. ca. $5 \%$ noch sehr gering ist. Dies wird sich zukünftig aufgrund der steigenden Fallzahlen schrittweise verändern. Verlässliche Ergebnisse sind deshalb erst in einigen Jahren zu erwarten, wenn Folgeerhebungen auch zeigen können, wie sich die Situation dieser Absolventengruppe im Berufsleben verändert. Eine weitere Einschränkung der Aussagekraft resultiert aus der unterdurchschnittlichen Beteiligung von Fachhochschulabsolventen, welche nur ca. $17 \%$ der Befragten stellen. Trotz aller Einschränkungen lassen sich auf der Ebene der Abschlussarten und der Hochschultypen dennoch belastbare Aussagen hinsichtlich einiger zentraler Aspekte wie der Entlohnung, der Niveauadäquanz der Beschäftigung und des fachlichen Bezugs zum Studienfach treffen, wie Tabelle 2 verdeutlicht (vgl. hierzu Alesi et al. 2010, S. 24ff.; Schomburg 2009, S. 59ff.).

Zunächst ist festzuhalten, dass eineinhalb Jahre nach dem Studienabschluss zwar 58 \% der Absolventen eines Bachelorstudiengangs an einer Fachhochschule, aber nur $20 \%$ der entsprechenden Absolventen von Universitäten den Einstieg in eine reguläre Berufstätigkeit gefunden hatten. Demgegenüber setzten $34 \%$ der Bachelorabsolventen an Fachhochschulen und sogar $72 \%$ an den Universitäten ihr Studium fort (Alesi et al. 2010, S. 31). Deutliche Unterschiede zwischen den Bachelorabsolventen von Universitäten und Fachhochschulen bestehen auch bei anderen Beschäftigungsaspekten. So entsprach der Anteil von Beschäftigten mit einem befristeten Arbeitsvertrag bei Fachhochschulabsolventen mit $30 \%$ dem Durchschnitt aller Abschlussarten, dieser Anteil lag aber für Universitätsabsolventen mit $45 \%$ deutlich darüber (ebd., S. 40). Auch beim Gehalt sind deutliche Unterschiede festzustellen: Dieses lag für die Gesamtgruppe der Bachelorabsolventen von Universitäten durchschnittlich um $20 \%$ unter dem Niveau der Absolventen von Diplom- und Magisterabschlüssen, bei den Absolventen von Bachelorstudiengängen an Fachhochschulen betrug die Differenz durchschnittlich $15 \%$ (ebd., S. 41). Der Wert für die Gruppe der Fachhochschulabsolventen ist insofern bemerkenswert, als der Bachelor von der Kultusministerkonferenz in ihren „ländergemeinsamen Strukturvorgaben für die Akkreditierung von Bachelor- und Masterstudiengängen“" explizit als Äquivalent zum bisherigen Fachhochschuldiplom definiert wurde (KMK 2010 und frühere Fassungen).

Auffällig sind weiterhin die Unterschiede zwischen den Abschlüssen hinsichtlich der Möglichkeiten zum Einsatz der im Studium erworbenen Qualifikationen. Über alle Abschlussarten hinweg gaben $43 \%$ der Befragten an, dass sie hierzu sehr gute oder gute Möglichkeiten haben; bei den Fachhochschulabsolventen sind es mit 39 \% etwas weniger. Von den Absolventen universitärer Bachelorstudiengänge berichten aber nur $27 \%$ über entsprechende Möglichkeiten zur Anwendung ihrer erworbenen Qualifikationen (Schomburg 2009, S. 87). Ebenfalls problematisch ist die Tatsache, dass nur $49 \%$ der Bachelorabsolventen der Universitäten ihre eigene berufliche Situation mit Blick auf die absolvierte Ausbildung als angemessen bewerten. Die Zustimmung der Absolventen von Bachelorstudiengängen an den Fachhochschulen liegt bei dieser Frage dagegen bei $61 \%$, was auch dem Durchschnitt aller Abschlussarten entspricht (ebd., S. 94). Mit den dargestellten Ergebnissen korrespondiert die unter den Absolventen von universitären Bachelorabschlüssen verbreitete Einschätzung, dass für ihre Tätigkeit kein Hochschulabschluss erforderlich wäre. Die Zustimmung zu dieser Aussage liegt mit $21 \%$ weit über dem Durchschnitt, der bei nur $8 \%$ liegt. Der Wert für Absolventen von Bachelorstudiengängen an Fachhochschulen liegt mit $12 \%$ etwas darüber (ebd., S. 90). Der Kern des Problems ist also nicht unbedingt, dass Bachelorabsolventen gar keine Beschäftigung finden würden. Dies ist den vorliegenden Daten zufolge nicht der Fall, was sich auch in der wachsenden Offenheit der Unternehmen für die neuen Abschlüsse widerspiegelt (vgl. z. B. Klös 2010). Aus einer „Employability“-Perspektive ließe sich die Studienstrukturreform also möglicherweise durchaus als Erfolg verbuchen. Diese Sichtweise wird aber den offenkundig bestehenden qualitativen Defiziten vieler Bachelorstudiengänge nicht gerecht. Schließlich sollte das Ziel einer qualifikationsadäquaten Beschäftigung ihrer Absolventen auch im vitalen Interesse der Hochschulen liegen.

Die Ergebnisse der INCHER-Absolventenstudie zeigen bei aller Vorläufigkeit deutlich, dass insbesondere für Absolventen des universitären Bachelors erheblich schlechtere Konditionen auf dem Arbeitsmarkt bestehen als bei den traditionellen Abschlüssen. Dies gilt sowohl bei der Befristung und dem Einkommen als auch bei der Adäquanz der Tätigkeit im Verhältnis zum Abschlussniveau, was darauf hindeutet, dass es für diese Studiengänge bislang nicht gelungen ist, ein eigenständiges berufsqualifizierendes Profil zu entwickeln und umzusetzen. $\mathrm{Zu}$ einem ähnlichen Ergebnis kommen Schaeper/Wolter (2008, S. 617) auf der Basis einer multivariaten Analyse des HIS-Absolventenpanels 2005. Demzufolge lassen sich im Hinblick auf fachspezifische Kompetenzen keine Unterschiede zwischen Fachhochschulabsolventen gestufter und traditioneller 
Studiengänge feststellen, während die Absolventen universitärer Bachelorstudiengänge sowohl ihre Fach- als auch ihre Methodenkompetenz signifikant schlechter einschätzen als die Absolventen traditioneller Diplom- und Magisterstudiengänge.

\section{Unbestimmter Begriff der akademischen Berufsqualifizierung}

Eine mangelnde Praxisorientierung vor allem der Studiengänge an Universitäten wird in der hochschulpolitischen Debatte seit vielen Jahren beklagt. Diese Einschätzung wird auch von den Studierenden selbst geteilt. Dem Studienqualitätsmonitor 2007 zufolge, der gemeinsam von HIS und der Konstanzer AG Hochschulforschung durchgeführt wurde, betrachten nur $39 \%$ der Studierenden an Universitäten den Praxisbezug ihres Studiums als gut oder sehr gut. Der entsprechende Wert bei den Fachhochschulstudierenden liegt mit $58 \%$ deutlich höher (Bargel et al. 2008, S. 21). Konsequenzen hat diese Differenz bei Eintritt in das Beschäftigungssystem. Hier werden von den Unternehmen traditionell überproportional viele Fachhochschulabsolventen und solche dualer Studiengänge bzw. von Berufsakademien eingestellt, weil sie auch aus Sicht der Unternehmen eine besonders praxisnahe Ausbildung durchlaufen haben (Pankow 2008). Der Deutsche Industrie- und Handelskammertag (DIHK) empfiehlt den Hochschulen deshalb eine verstärkte Einbindung von Praxisanteilen in ihre Studiengänge. Ähnliche Plädoyers sind in der hochschulpolitischen Debatte zahlreich zu finden. So zum Beispiel im „Employability-Rating“ des Centrums für Hochschulentwicklung (CHE) und des Arbeitskreises Personalmarketing (dapm), das im Jahr 2008 für betriebswirtschaftliche und ausgewählte technische Studiengänge durchgeführt wurde. Hierbei wurde die Vermittlung zentraler beruflicher Kompetenzen unmittelbar aus der Studienstruktur abgeleitet. Beispielsweise bildeten in der Dimension Praxisbezug die Existenz eines CareerCenters an der jeweiligen Hochschule und die Länge der obligatorischen Praxisphase die zentrale Bewertungsgrundlage (CHE o.J. (2008)). Aus dem Anteil als praxisrelevant deklarierter Lehrveranstaltungen im vorgegebenen Curriculum unmittelbar auf die erfolgreiche Vermittlung von Kompetenzen zu schließen, ist aber empirisch schwer haltbar, da die Aneignung präsentierten Wissens einen aktiven Lernprozess der Studierenden voraussetzt, der von vielen verschiedenen Einflussfaktoren abhängt (z. B. Wildt 2007).

Hinzu kommt, dass die inhaltliche Ausgestaltung eines „Berufsbezugs“ für die akademische Ausbildung alles andere als einfach ist, da sich ein klares Berufsbild für Studiengänge nur in den seltensten Fällen definieren lässt. Auf der Grundlage der Daten des Mikrozensus' von 1995 bis 2005 hat das IW deutlich gemacht, dass Hochschulabschlüsse die Grundlage für einen sehr breiten beruflichen Einsatz bilden. So arbeiten beispielsweise lediglich $59 \%$ der ingenieurwissenschaftlichen Absolventen in Ingenieurberufen (Anger/KonegenGrenier 2008, S. 11f.). Insgesamt, so die Schlussfolgerung des IW, sei die Beziehung von studierter Fachrichtung und ausgeübtem Beruf als flexibel zu betrachten. Die im Studium erworbenen Fähigkeiten und Kenntnisse verlören somit auch bei einem Berufswechsel nicht an Verwertbarkeit. Praxisorientierung als Bildungsziel der neuen Studiengänge kann folglich nicht bedeuten, dass die Studieninhalte auf ein eng definiertes Berufsbild ausgerichtet sein sollten. Die Befunde des IW können vielmehr als Signal gegen eine zu hohe Spezialisierung verstanden werden.

\section{7 \\ Berufs- und Praxisbezug als mögliche Alternativen}

Die Vorbereitung auf eine berufliche Tätigkeit ist nicht die einzige Zielsetzung eines Studiums. Koepernik/Wolter (2010, S. 60f.) formulieren drei weitere Ziele der akademischen Ausbildung:

- das Verständnis wissenschaftlicher Methoden, Theorien und Wissensbestände und der kritische Umgang mit diesen,

- die Ausbildung einer spezifisch wissenschaftlichen Haltung, die auch eine ausgeprägte Reflexionsfähigkeit umfasst, und - die Vermittlung von Schlüsselkompetenzen.

Durch das Zusammenwirken dieser Aspekte könnten Absolventen eine „wis- senschaftsbasierte, professionelle Handlungskompetenz" erwerben (ebd., S. 61). Aus Sicht von Koepernik/Wolter ist dieser Begriff besser als beispielsweise der „Employability“-Begriff dafür geeignet, die multiplen Ziele eines Studiums zu beschreiben, da nicht allein die Aufnahme eines Beschäftigungsverhältnisses nach dem Studienabschluss im Fokus stehen dürfe, sondern mit einem wissenschaftlichen Studium auch ein qualitativer Anspruch verbunden sei, der sich sowohl an die erworbene Kompetenz als auch an die Art der Beschäftigung richten müsse.

Mit der Forderung nach einer stärkeren Arbeitsmarktorientierung von Studium und Lehre werden zudem unterschiedlichste Studienkonzepte verbunden. Schindler (2004, S. 7) fasst die verschiedenen Strömungen der hochschulpolitischen Diskussion zu drei Grundmodellen zusammen:

(1) Eine Praxisorientierung der Studiengänge, die eine „Bezugnahme und Vorbereitung der Studierenden auf die allgemeine berufliche Praxis“ beinhaltet, „ohne dass bestimmte Berufe den Bezugspunkt darstellen".

(2) Eine Berufsorientierung, die „den Erwerb fachlich-inhaltlicher, methodischer und sozialer Qualifikationen für Tätigkeiten in einem speziellen berufsspezifischen Aufgabenspektrum“ meint, wobei sich das Studium an „breite(n) berufliche(n) Tätigkeitsfelder(n)“ orientieren soll.

(3) Die Employability, die die Fähigkeit einer Person bezeichnet, „auf der Grundlage ihrer fachlichen und Handlungskompetenzen, Wertschöpfungs- und Leistungsfähigkeit ihre Arbeitskraft anbieten zu können und damit in das Erwerbsleben einzutreten, ihre Arbeitsstelle zu halten oder, wenn nötig, sich eine neue Erwerbsbeschäftigung zu suchen" (Blancke et al. 2000, S. 9, zitiert nach: Schindler 2004, S. 6).

Dieser Typologie zufolge stehen bei der Praxisorientierung die fachlichen Inhalte im Vordergrund des Studienkonzepts, während der Schwerpunkt bei der Employability bei der Vermittlung von Schlüsselkompetenzen liegt und die Disziplin in den Hintergrund rückt. Das Konzept der Berufsorientierung nimmt eine mittlere Position ein. Empirische Studien zeigen 
aber, dass es bei der Konzeption von Studiengängen kein „Tauschgeschäft zwischen Schlüssel- und Fachkompetenzen geben kann", da erst Schlüsselkompetenzen und Fachkompetenzen gemeinsam eine professionelle Handlungskompetenz ermöglichen (Schaeper/Wolter 2008, S. 621). Weiterhin weist etwa Teichler (2008, S. 70) darauf hin, dass eine stärkere Praxisorientierung auch von denjenigen gefordert wird, die hierunter eine Ergänzung der unabhängigen wissenschaftlichen Grundlegung und humanistischen Persönlichkeitsförderung durch berufsnützliche Befähigungen, durch Informationen über den Arbeitsmarkt und Beruf sowie durch beruflich wertvolle Werthaltungen und Verhaltensstile verstehen (vgl. zur Debatte auch: Arbeitsgruppe Alternative Wirtschaftspolitik 2006, S. 105ff.; Wildt 2007; Banscherus et al. 2007).

Die Ergebnisse der zitierten INCHERAbsolventenstudie zeigen, dass es den Fachhochschulen offensichtlich besser gelingt, ihren Studierenden in Verbindung mit den fachlich-wissenschaftlichen Inhalten arbeitsmarktrelevante Kompetenzen zu vermitteln. Eine Ursache hierfür dürfte die traditionell hohe Berufsorientierung der Fachhochschulen sein, die sich aufgrund des ähnlichen strukturellen und curricularen Rahmens von Diplom- und Bachelorabschlüssen auch auf die gestufte Studienstruktur übertragen lässt. Demgegenüber haben die Universitäten vielfach auf eine weitgehend unverbundene Parallelität von Fach- und Schlüsselkompetenzen im Sinne des Employability-Ansatzes gesetzt. Die empirischen Daten belegen eindrücklich, dass dieser Weg nicht dazu geeignet ist, ein eigenständiges berufsqualifizierendes Profil des universitären Bachelorabschlusses zu definieren. Erfolg versprechender dürften unter Berücksichtigung der spezifischen Inhalte und Anforderungen des Studienfachs die Ansätze der Praxis- bzw. Berufsorientierung sein.

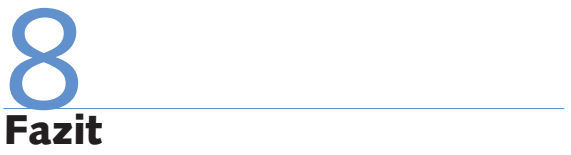

Insgesamt werfen die bisherigen Ergebnisse empirischer Studien mehr Fragen auf als sie Antworten geben können, da die Fallzahlen für Bachelor- und Masterstudiengänge bei den Absolventenstudien von HIS und INCHER aktuell noch zu gering sind. Aufgrund dessen sind auch Differenzierungen nach Fächergruppen nicht sinnvoll vorzunehmen. So ist eine eindeutige Bewertung des Übergangs von Absolventen der neuen Studiengänge in den Arbeitsmarkt auf der Grundlage der bisherigen Daten noch nicht möglich. Es wird allerdings dennoch deutlich, dass insbesondere beim universitären Bachelor noch viele Unklarheiten und Probleme bestehen, da die Gesamtschau der einzelnen Indikatoren wie Übergangsverhalten, Vertragssituation, Einkommenshöhe und Qualifikationsadäquanz der Tätigkeit ein recht eindeutiges - unbefriedigendes Bild ergibt. Dies betrifft allerdings nicht nur die Einordnung des Abschlusses auf dem Arbeitsmarkt, sondern geht weit in die Konzeption und die Durchführung der Studiengänge hinein. Die Unternehmensbefragungen des IW sowie die Studien von Schaeper/Wolter (2008), Dobischat et al. (2008) sowie Koepernik/Wolter (2010) zeigen weiterhin, dass die Antwort auf die Frage, zu welchem fachlichen Wissen und zu welchen übergreifenden Kompetenzen ein Bachelorstudium führen soll, bislang unklar ist. Gleiches gilt für die Position, die der Bachelor im gewachsenen Qualifikationsgefüge einnehmen soll. Nach wie vor ist nicht geklärt, wie er sich von den Abschlüssen der beruflichen Aus- und Fortbildung sowie vom Masterabschluss und den traditionellen Hochschulabschlüssen abgrenzen kann. Weitergehende Forschungsarbeiten sollten deshalb nicht nur den Übergang in ein Beschäftigungsverhältnis zum Gegenstand ihrer Untersuchungen machen, sondern stärker qualitative Aspekte wie die Angemessenheit der Beschäftigung und die Verbindung von Berufsvorbereitung und Fachstudium in die Betrachtung einbeziehen. Politisch opportune, aber vor dem Hintergrund der Datenlage eher pflichtschuldig wirkende Phrasen wie „Bachelor welcome“ oder „Bachelor-geht doch “ können die bestehende inhaltliche Leere sicherlich nicht füllen. Eine Orientierung für eine praxistaugliche Konzeption der neuen Studiengänge könnten die Modelle der Praxis- und der Berufsorientierung bieten, die aufgrund ihrer Orientierung an einer umfassenden Handlungskompetenz gegenüber einem eng geführten Employability-Ansatz im Vorteil sind, indem sie eine Verbindung von Elementen der Arbeitswelt mit akademischen Anforderungen ermöglichen.

\section{LITERATUR}

\begin{abstract}
Alesi, B./Schomburg, H./Teichler, U. (2010): Humankapitalpotenziale der gestuften Hochschulabschlüsse in Deutschland. Weiteres Studium, Übergang in das Beschäftigungssystem und beruflicher Erfolg von Bachelor- und Master-Absolventen, Studien zum deutschen Innovationssystem 13, Berlin
\end{abstract}

Anger, C./Konegen-Grenier, C. (2008): Die Entwicklung der Akademikerbeschäftigung, IW-Trends 1, Köln

Arbeitsgruppe Alternative Wirtschaftspolitik (Hrsg.) (2006): Memorandum 2006. Mehr Beschäftigung braucht eine andere Verteilung, Köln
Bake, W. T. et al. (2006): More Bachelors and Masters welcome! Reformen konsequent umsetzen - Ausbildungsqualität deutlich steigern. Erklärung der Personalvorstände führender Unternehmen in Deutschland zur Hochschulreform vor dem Hintergrund steigender Studierendenzahlen, 30.05., Berlin

Banscherus, U./Gulbins, A./Himpele, K./Staack, S. (2009): Der Bologna-Prozess zwischen Anspruch und Wirklichkeit. Die europäischen Ziele und ihre Umsetzung in Deutschland. Eine Expertise im Auftrag der Max-Traeger-Stiftung, Frankfurt am Main 
Banscherus, U./Schewe, L./Staack, S. (2007): Praxisorientierung als Studienreform, in: Bund demokratischer Wissenschaftlerinnen und Wissenschaftler/freier zusammenschluss von studentInnenschaften/ Gewerkschaft Erziehung und Wissenschaft (Hrsg.): Bildung - Beruf Praxis. Bildungsreform zwischen Elfenbeinturm und Verwertungslogik, Marburg, S. 36-38

Bargel, T./Müßig-Trapp, P./Willige, J. (2008): Studienqualitätsmonitor 2007. Studienqualität und Studiengebühren, Hannover

Bargel, T./Ramm, M./Multrus, F. (2008): Studiensituation und studentische Orientierungen. 10. Studierendensurvey an Universitäten und Fachhochschulen, Bonn/Berlin

Bensel, N. et al. (2004): Bachelor welcome! Erklärung führender deutscher Unternehmen zur Umstellung auf Bachelor- und Masterabschlüsse in Deutschland, 07.07., Berlin

Briedis, K. (2007): Übergänge und Erfahrungen nach dem Hochschulabschluss. Ergebnisse der HIS-Absolventenbefragung des Jahrgangs 2005, Hochschulinformationssystem (HIS), Forum Hochschule 13, Hannover

Bundesministerium für Bildung und Forschung (BMBF) (2009): Schavan: „Bologna-Reform gemeinsam weiterentwickeln“. Bundesministerin will Anregungen der Studierenden aufgreifen, Pressemitteilung vom 07.07., Berlin

Centrum für Hochschulentwicklung (CHE) (o.J. (2008)): CHE-/dapmEmployability-Rating, http://www.che-ranking.de/cms/?getObject= 527\&getLang=de (26.02.2010)

Deutscher Industrie- und Handelskammertag (DIHK) (2004): Fachliches Können und Persönlichkeit sind gefragt. Ergebnisse einer Umfrage bei IHK-Betrieben zu Erwartungen der Wirtschaft an Hochschulabsolventen, Berlin

Dobischat, R./Fischel, M./Rosendahl, A. (2008): Auswirkungen der Studienreform durch die Einführung des Bachelorabschlusses auf das Berufsbildungssystem. Eine Problemskizze, Düsseldorf

Europäische Bildungsminister (1999): Der Europäische Hochschulraum. Gemeinsame Erklärung der Europäischen Bildungsminister,

19. Juni, Bologna

Europäische Bildungsminister (2003): „Den Europäischen Hochschulraum verwirklichen“ - Kommuniqué der Konferenz der

europäischen Hochschulministerinnen und -minister am 19. September, Berlin

Europäischer Rat (2000): Schlussfolgerungen des Vorsitzes, 23. und 24. März, o.O. (Lissabon)

Geighardt, C. (2009): Befragungsergebnisse der Deutschen Gesellschaft für Personalführung (DGFP e.V.) zum Thema "Bachelor welcome!?", Personalblitzlicht 1, Düsseldorf

Habermann, W./Lohaus, D. (2006): Bachelor ante portas, in: Personal - Zeitschrift für Human Resource Management 3, S. 6-8

Heine, C./Willich, J./Schneider, H./Sommer, D. (2008): Studienanfänger im Wintersemester 2007/08. Wege zum Studium, Studien- und
Hochschulwahl, Situation bei Studienbeginn, Hochschulinformationssystem (HIS), Forum Hochschule 16, Hannover

Hochschulrektorenkonferenz (HRK) (2009): Statistische Daten zur Einführung von Bachelor- und Masterstudiengängen. Sommersemester 2009, Statistiken zur Hochschulpolitik 1, Bonn

Klös, H.-P. (2010): Neue Signale für den Arbeitsmarkt - Zur Akzeptanz gestufter wissenschaftlicher Abschlüsse in der betrieblichen Praxis, in: WSI-Mitteilungen 5, S. 257-262

Koepernik, C./Wolter, A. (2010): Studium und Beruf, Arbeitspapier 210 der Hans-Böckler-Stiftung, Düsseldorf

Konegen-Grenier, C. (2004): Akzeptanz und Karrierechancen von Bachelor- und Masterabsolventen deutscher Hochschulen, IW-Trends 2, Köln

Konegen-Grenier, C./Koppel, O. (2009): Akzeptanz und Karrierechancen von Ingenieuren mit Bachelor- oder Masterabschluss,

IW-Trends 4, Köln

Krawietz, M. (2007): Bachelor! Was sonst? Abschlussarten deutscher Studienanfänger im Wintersemester 2006/2007, Hannover

Kultusministerkonferenz (KMK) (2010): Ländergemeinsame Strukturvorgaben gemäß §9 Abs. 2 HRG für die Akkreditierung von Bachelorund Masterstudiengängen - Beschluss der Kultusministerkonferenz vom 10.10.2003 i.d.F. vom 04.02.2010

Pankow, F. (2008): Die Studienreform zum Erfolg machen! Erwartungen der Wirtschaft an Hochschulabsolventen, Berlin

Schaeper, H./Wolter, A. (2008): Hochschule und Arbeitsmarkt im Bologna-Prozess. Der Stellenwert von „Employability“ und Schlüsselkompetenzen, in: Zeitschrift für Erziehungswissenschaft 4, S. 607-625 Schindler, G. (2004): Employability und Bachelor-Studiengänge eine unpassende Verbindung, in: Beiträge zur Hochschulforschung 4, S. $6-26$

Schomburg, H. (2009): Generation Vielfalt. Ausgewählte Ergebnisse des Projekts "Studienbedingungen und Berufserfolg. Befragung Jahrgang $2007^{\prime \prime}$, Kassel

Teichler, U. (2008): Der Jargon der Nützlichkeit. Zur EmployabilityDiskussion im Bologna-Prozess, in: Das Hochschulwesen, Forum für Hochschulforschung, -praxis und -politik 03, S. 68-79

Werner, D./Hollmann, C./Schmidt, J. (2008): Wie entwickeln sich angesichts des Strukturwandels zur Wissensgesellschaft und der Einführung der Bachelorstudiengänge die Chancen für duale Ausbildungsberufe und das duale System? Abschlussbericht des Instituts der deutschen Wirtschaft Köln an das Bundesministerium für Wirtschaft und Technologie, Köln

Wildt, J. (2007): Praxisbezug in Studium und Lehre. Anmerkungen aus Sicht der Hochschuldidaktik, in: Bund demokratischer Wissenschaftlerinnen und Wissenschaftler/freier zusammenschluss von studentInnenschaften/Gewerkschaft Erziehung und Wissenschaft (Hrsg.): Bildung - Beruf - Praxis. Bildungsreform zwischen Elfenbeinturm und Verwertungslogik, Marburg, S. 32-35 